\title{
EXPERIÊNCIA NO TRATAMENTO DOMICILIAR EM PATOLOGIAS ORTOPÉDICAS: Atuaçāo da Enfermeira no Ambulatório de Ortopedia Pediátrica.
}

\author{
Maria do Carmo Barretto de Carvalho *
}

\begin{abstract}
RESUMO - A autora se propõe a mostrar a atuação direta da enfermeira na realização, orientação e controle do tratamento ambulatorial e domiciliar nas crianças portadoras de Luxação Congênita do Quadril, Doença de Legg-Calvé-Perthes e Sinovite Transitória do Quadril.
\end{abstract}

ABSTRACT - The author intends to show the nurses' performance in
hometreatment in orthopedics' diseases, through a preparation of traction
or abduction diaper, involving 21 patients.

\section{INTRODUÇĀO}

Atualmente, a decisão de se manter pacientes internados em hospitais, tem levado em consideração o risco de infecção hospitalar, condições físicas e psicológicas do paciente e o custo deste tipo de tratamento. Isto ocorre especialmente em patologias ortopédicas, onde a duração do tratamento $e$, por vezes, considerada longa e dispendiosa tanto para o paciente quanto para o hospital, conforme levantamento realizado no Hospital Sarah.

Dentre as patologias ortopédicas infantis que requerem tratamento mais complexo e prolongado, 04 (quatro) apresentam maior freqüência, quais sejam: Pé Equino Varo Congênito, Luxação Congênita do Quadril, Doença de Legg-Calvé-Perthes e Sinovite Transitória do Quadril.

No SARAH, a atuação da enfermeira do Ambulatório verifica-se na ortopedia infantil, através da orientação de confecção da fralda de abdução (ou travesseiro de Frejka) em Luxação Congênita do Quadril e da montagem da tração domiciliar em Legg-Calvé-Perthes e Sinovite Transitória do Quadril. Estes dois tipos de tratamento têm a finalidade de manter o quadril luxado em posição de abdução, no caso da fralda, e, na tração, manter o paciente em repouso. E quando há necessidade de internação hospitalar, verificamos que com freqüência impõe à famńlia envolvida uma readaptação, às vezes traumática, à nova situação. Segundo $\mathrm{CHAPMAN}^{4}$ e GELLERT $^{7}$ problemas psicológicos e desentendimentos familiares têm sido reconhecidos e relatados com certa freqüência.

Assim, os objetivos deste estudo compreendem: analisar o efeito do tratamento domiciliar, a repercussão psicológica à criança através da opinião dos pais e demonstrar a atuação direta da enfermeira.

\section{DESEN VOL VIMENTO}

No período de julho de 1985 a maio de 1989, foram analisados 21 (vinte e um) pacientes residentes no Distrito Federal. Utilizou-se para a coleta de dados: aplicação de questionário (vide anexo II), análise de prontuários e entrevista com os pais.

\section{Tratamento Ambulatorial}

Fralda de abduçāo - a fralda de abdução é indicada em pacientes de 0 a 2 anos de idade, portadores de Luxação Congênita do Quadril, pois tem a finalidade de manter o quadril em posição estável de flexão e abdução, permitindo alguns movimentos ativos e evitando maiores complicações do quadril que está luxado. De acordo com BARLOW ${ }^{1}$, DONAHOO ${ }^{6}$, POWELL $^{13}$ e SAHLSTRAND ${ }^{15}$, é necessário o tratamento precoce, se ja de qual tipo for.

Após a fase inicial do tratamento dos pacientes portadores de Luxação Congênita do Quadril, quando é feita redução da cabeça femural no acetábulo, os ortopedistas utilizam a fralda de abdução para manutenção desta redução. A confecção, colocação e controle do uso da fralda de abdução é orientado pela enfermeira no ambulatório.

A enfermeira então, mostra aos pais um modelo da fralda de abdução e mede a distância entre os joelhos da criança, na posição de abdução e flexão dos quadris e também do comprimento do tronco, desde a linha mamilar até a região dorsal, contornando o períneo.

Utiliza-se preferencialmente o tecido de algodão, de maneira a facilitar as trocas de fraldas, sendo de fácil limpeza e adequado ao clima tropical. Para firmeza e sustentação, coloca-se internamente uma espuma de $3 \mathrm{~cm}$ de espessura. (Vide anexo 2, fig. 1).

* Enfermeira do Hospital das Doenças do Aparelho Locomotor - SARAH - Brasília-DF 
Após a confecção da fralda, os pais retornam ao ambulatório para que a enfermeira confirme as medidas, o correto posicionamento da criança e oriente quanto ao uso e a importância desşe tipo de sutese.

\section{Tratamento Domiciliar}

No tratamento das crianças com Doença de Perthes, no Sarah, é utilizado protocolo de tratamento que visa manter a amplitude de movimentos do quadril afetado. Segundo este protocolo, quando o paciente apresenta abdução igual ou inferior a 20 graus, é necessário o uso de tração cutânea, pelo período de 2 semanas, após o que, se houve aumento da abdução, o paciente é orientado a realizar exercícios para reforço muscular e manutenção da amplitude articular obtida. Caso a tração não seja efetiva, é necessário a cirurgia de tenotomia dos adutores da coxa, seguida de gesso.

Além dos pacientes com necrose avascular da epífise proximal do fêmur, Doença de Perthes, também é frequiente observarmos em nosso ambulatório crianças com limitação dos movimentos, dor no quadril e claudicação, sem as alterações radiológicas e clínicas que caracterizam a Doença de Perthes ou outras patologias do quadril. O quadro descrito, caracteriza a assim denominada Sinovite Transitória do Quadril, onde a conduta é a instalação de tração cutânea para repouso durante 2 semanas, após o que, ocorre na maioria dos casos a remissão dos sintomas.

Traçāo cutânea de Buck - este tipo de tração é indicado quando se faz necessário repouso, como em Legg-Calvé-Perthes e Sinovite Transitória do Quadril, segundo CATTERALL ${ }^{3}$, KLISIC $^{10}$ e GERSHUNI ${ }^{8}$.

O princípio da tração aplicada ao corpo humano, pode ser entendido como uma força externa e contínua aplicada sobre um segmento corporal na tentativa de neutralizar a contratibilidade muscular. A tração cutânea atua indiretamente sobre o osso, uma vez que é aplicada sobre a pele. No entanto, esta tração não suporta quantidade elevada de peso e é contra-indicada na presença de lesões de pele.

Em função da necessidade de separar a criança de sua famnlia para a realização de tração no hospital, e das repercussões psicológicas que isto acarreta tanto na criança como na famnia, desde a década de 70, passamos a utilizar a tração domiciliar, que é realizada e controlada pela enfermeira do ambulatório.

É função da Enfermeira:

- Verificar o peso da criança, fazendo a mensuração nos membros inferiores, do ponto acima do maléolo medial até o ponto $1 / 3$ proximal da coxa;

- Solicitar ao pais a arrumação do quarto da criança (informando-se a respeito das características da cama), uma tábua de madeira $(30 \mathrm{~cm}$ de altura $\times 2 \mathrm{~cm}$ de espessura $x 1,50 \mathrm{~cm}$ de comprimento) uma furadeira elétrica e a confecção de um short aberto dos lados para simplificar a realização da necessidade fisiológica na cama;

- Marcar o dia e a hora de visita domiciliar

a) Material necessário à tração:

- um par de blocos de espuma;

- ataduras de crepom;

- fita crepe;

- malha tubular;

- suporte para peso;

- ganchos;

- fio de nylon;

- pesos (10\% do peso corporal);

- fita métrica;

- tesoura.

b) Técnica de confeção de tração (vide anexo 2 , fig. 2):

- posicionar o indivíduo em decúbito dorsal com os membros inferiores em abdução máxima e rotação interna dos pés;

- enrolar as ataduras de crepom em ambos os membros;

- posicionar a espuma, passar as ataduras de crepom e enrolar a malha tubular;

- adaptar ao suporte de tração o fio de nylon que passa pelos orifícios realizados na tábua presa aos pés da cama. A posição desses orifícios é determinada pelo grau de abdução desejado na tração. Na outra extremidade do fio é então colocado os ganchos e os suportes com os pesos determinados (10\% do peso corporal).

c) Orientação aos pais:

- correto posicionamento, ou seja, abdução máxima dos membros inferiores, podendo a criança permanecer sentada ou deitada na cama;

- cuidado com a pele, observando a temperatura, cor e textura, atentando para qualquer intercorrência, principalmente queixa de dor;

- higiene corporal - banho no chuveiro e aplicação de óleo e/ou creme na pele antes de montar novamente a tração. Necessidades fisiológicas na cama, sendo que se necessário, levar a criança ao banheiro;

- observação do posicionamento dos pesos, pois estes não podem estar apoiados ao solo e devem estar simétricamente alinhados;

- alimentação da criança deve ser oferecida na cama e preferencialmente baseada em alimentos leves e laxantes; a ingestão de líquidos deve ser estimulada;

- a recreação deve ser cuidadosamente esti- 
mulada e se a criança estiver em idade escolar, mantem-se o ensino e tarefas;

- a consulta ambulatorial pós-tração fica remarcada e os pais devem trazer o material utilizado para o hospital

\section{RESULTADOS}

Do total de 21 pacientes, na faixa etária de 4 a 10 anos, $16(76,21 \%)$ eram do sexo masculino e $5(23,8 \%)$ do feminino (Vide Anexo 3, tabela 1). Todos os pacientes residiam no Distrito Federal, sendo que $12(57,1 \%)$ residiam em alguma cidade satélite (região periférica de Brasília) e $9(42,9 \%)$ no piloto (região central).

A duração da tração foi de 15 dias em média, sem variações significantes em função do diagnóstico.

Para análise da efetividade, os pacientes foram divididos em dois grupos, segundo $o$ diagnóstico: grupo I - pacientes portadores de Legg-Calvé-Perthes; grupo II - pacientes portadores de Sinovite Transitória do Quadril. Verificamos que do total de pacientes analisados, 12 $(57,1 \%)$ pertencem ao grupo I e $9(42,9 \%)$ ao grupo II. (Vide anexo 3, Tabela 2).

Na Tabela 2 (Anexo 3), o grupo I apresentou $9(75 \%)$ resultados ef etivos contra $3(25 \%)$ não efetivos. Entretanto, o grupo II apresentou maior efetividade, $8(88,9 \%)$ contra $1(11,1 \%)$ não efetivo.

Dos pais e/ou responsáveis entrevistados 34 (100\%) consideraram bom o tratamento realizado.

\section{DISCUSSĀO}

Na Luxação Congênita do Quadril o objetivo do tratamento é reduzir a luxação mantendo sua redução e preservando as funções do quadril. Diferentes ortopedistas adotam diversas técnicas e, conservador ou cinúrgico, o melhor tratamento é, considerado por DERQUI ${ }^{5}, \mathrm{PO}-$ WELL $^{13}$ e SAHLSTRAND ${ }^{15}$, aquele iniciado o mais cedo possível. No Sarah, adotamos primeiramente o tratamento conservador e caso este não se ja satisfatório, optamos pelo cirúrgico.

Dentre os tipos de tratamento conservador, fralda de abdução é utilizada freqüentemente, tão logo a criança (de 0 a 2 anos) seja avaliada pelo ortopedista.

A tração realizada no domicńlio do paciente foi primeiro relatada por $\mathrm{BOOS}^{2}$, seguida de KEENAN $^{9}$, MUBARAK ${ }^{12}$ e PREUSS ${ }^{14}$ com o intuito de diminuir gastos hospitalares e reduzir problemas decorrentes da separação da criança e pais durante o tratamento da Luxação Congênita do Quadril.

No nosso estudo, a efetividade do tratamento por tração foi de $80,9 \%$ (17 pacientes) contra $19,1 \%$ (4 pacientes) não ef etivos.
Para a Doença de Perthes verificou-se eficácia da tração domiciliar em 9 (75\%) crianças, sendo que nos 3 pacientes em que não houve aumento da amplitude articular foi repetida a tração por mais 15 dias no hospital, com a solução do problema em dois casos. Um dos pacientes, mesmo com a tração no hospital, não apresentou melhora, sendo necessário o procedimento cirúrgico. Dentre as 9 crianças com o diagnóstico de Sinovite Transitória do Quadril, a tração foi eficiente em 8 (88,9\% dos casos). foram

As principais razões da falha do método,

- Fálta de observação dos pais para a manutenção da tração, permitindo que os pesos ficassem apoiados no chão, não atentando para o correto posicionamento da criança e até mesmo permitindo que a criança ficasse fora da tração.

- Não aceitação do procedimento por parte da criança, que nesta faixa etária apresenta uma natural hiperatividade, e, estando restrita ao leito, não pode realizar suas atividades lúdicas.

- Fatores inerentes à própria patologia, tais como deformidades estruturadas que necessitaram de tratamento complementar à tração.

A opinião dos pais foi favorável ao método em $100 \%$ dos casos, pois segundo afirmaram, a criança tratada no domicńlio sofre menos transtornos psicosociais, justamente porque seu ambiente terapêutico é conhecido e nao ocorre a separação familiar, confirmando o que relata CHAPMAN $^{4}$ e GELLERT ${ }^{7}$.

A visita domiciliar realizada pela Enfermeira, orientando e analisando as possibilidades de manter crianças e/ou adultos em tratamento domiciliar, reforça ainda mais a importância da saúde pública hospitalar, de acordo com LORI$\mathrm{CH}^{11}$ e SEDDON ${ }^{16}$, pois esta tem como objetivo o paciente tratado (curativo e preventivamente) em seu domicílio. Ainda segundo KEENAN $^{9}$ e MUBARAK ${ }^{12}$ a enfermeira é a chave do sucesso do tratamento domiciliar, pois através de sua orientação e conhecimento teórico-prático é que a família e a criança envolvida compreendem os benefícios do tratamento e participam ativamente de sua execução.

\section{CONCLUSĀO}

Consideramos que a atuação da enfermeira no ambulatório de ortopedia pediátrica, se ja na orientação e controle da confecção e uso de orteses para a lux̣ação congênita, seja ná execução do método de tração domiciliar, propicia melhor aceitação do tratamento pelo paciente e 
pela famnlia e reduz as repercussōes psicológicas, sempre presentes, nos tratamentos ortopédicos em crianças.

Assim, em função desses resultados, é nossa impressão que o tratamento domiciliar deve ser considerado, sempre que possível, no tratamento das patologias ortopédicas em crianças, sendo fundamental a participação efetiva da enfermeira.

\section{REFERÊNCIAS BIBLIOGRÁFICAS}

1 BARLOW, T.G. Early diagnosis and treatment of congenital dislocation of the hip. J. Bone. Joint. Surg., 44 (B 2): 292-301, may, 1962.

2 BOOS, M.L. A program of home traction for congenital dislocation of the hip. Orthop. Nurs., 2: 11-6, 1982.

3 CA T TERALL, A. Legg-Calvé-Perthes Syndrome. Clin. Orthop., 158: 41-52, july/aug., 1981.

4 CHAPMAN, A.H., LOEB, D.G., GIBBONS, M.J. Psychiatric aspects of hospitalizing children. Arch. Pediat., $73: 77,1965$.

5 DERQUI, Juan Cruz. Patogenia y tratamiento de la Luxacion Congenita de la cadera.Bol.y Trab.dela S.A.O.T Āno XXXIII, 7: 1-23, noviembre, 1973.

6 DONAHOO, Clara A. Enfermagem em Ortopedia e Traumatologia. Trad. de Antonio A. lberto Serra. Editora Pedagógica e Universitária, São Paulo, 1977, 288p.

7 GELLER $\Gamma$, E. Reducing the emotional stresses of hospitalization for children. Am. J. Occup. Ther., 12:125, 1958.

8 GERSHUNI, David H. et alii. Arthrographic findings in Legg-Calvé-Perthes Disease and Transient Synovitis of the hip. J. Bone. Joint. Surg., 60 A (4): 457-464, june, 1978.
9 KEENAN, J. et alii. Home traction in the management of congenital dislocation of the hip. Clin. Orthop., 165: 83-90, may, 1982.

10 KLISIC, P.J. et alii. Perthes' disease. Int. Orthop., 8 (2): 95-102, 1984.

11 LORICH, M.L. Nurses provide more than health care. Migrant workers clinic. Child today, 14(4): 30-4, jul/aug, 1985 .

12 MUBARAK, S.J. et alii. Home traction in. the managemen of congenital dislocation of the hips. Journal of Pediatric Orthopedics, 6(6): 721-723, New York, 1986.

13 POWELL, Mary. Orthopaedic Nursing and Rehabititation. 9. ed. Edinburgh: E. \& S. Livingstone, 1962, 516p.

14 PREUSS, Antonio Osny et alii. Luxação congênita do quadril: resultado do tratamento conservador (grupo etário 6-24 meses). Revista Brasileira de Ortopedia, 23(7): 197-204, julho, 1988 .

15 SAHLS IRAND, $T$. et alii. Management of neonatal hip instability: an analysis of the efficiency in a consistent treatment program. Journal of Pediatric Orthopedics, 5: 540-545, New York, 1985.

16 SEDDON, T.D. et alii. Practice rurse home visits survey Otumoetai Health Center. NZ Med. J., 97(748): 45-7, jan, 1985.

ANEXO 1

QUESTIONÁRIO

I - Características do paciente

Nome:

Registro:

Idade:

Sexo: ( ) masculino ( ) feminino

Procedência: ( ) Plano Piloto

) Cidade Satélite

II - Características da patologia

Patologia ( ) Luxação Congênita do Quadril

) Legg - Calvé - Perthes

( ) Sinovita Transitória do Quadril

( ) Outros

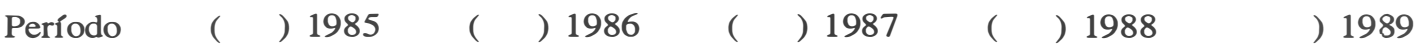

Tratamento domiciliar

a) Tipo: ( ) tração cutânea Buck

) fralda de abdução

b) Duração: ( $\quad \begin{aligned} & 1 \text { semana } \\ (\quad & \text { ) } 2 \text { meses } \\ (\quad & 5 \text { meses }\end{aligned}$

( ) 2 semanas

( ) 1 mês

(. ) 3 meses

( ) 4 meses

58 R. Bras. Enferm., Brasília, 44 (4): 55-60, out./dez. 1991 

c) Resultado: ( ) efetivo
( ) não efetivo
d) Por quê?
e) Tratamento Hospitalar posterior ao Domiciliar. ( ) sim ( ) não
f) alta:

$$
\begin{aligned}
& \text { ( ) sim ( ) em tratamento } \\
& \text { ( ) não con pareceu à última consulta }
\end{aligned}
$$

III - Opinião dos pais

$$
(\text { ) bom ( ) regular ( ) insatisfatório }
$$

ANEXO 2

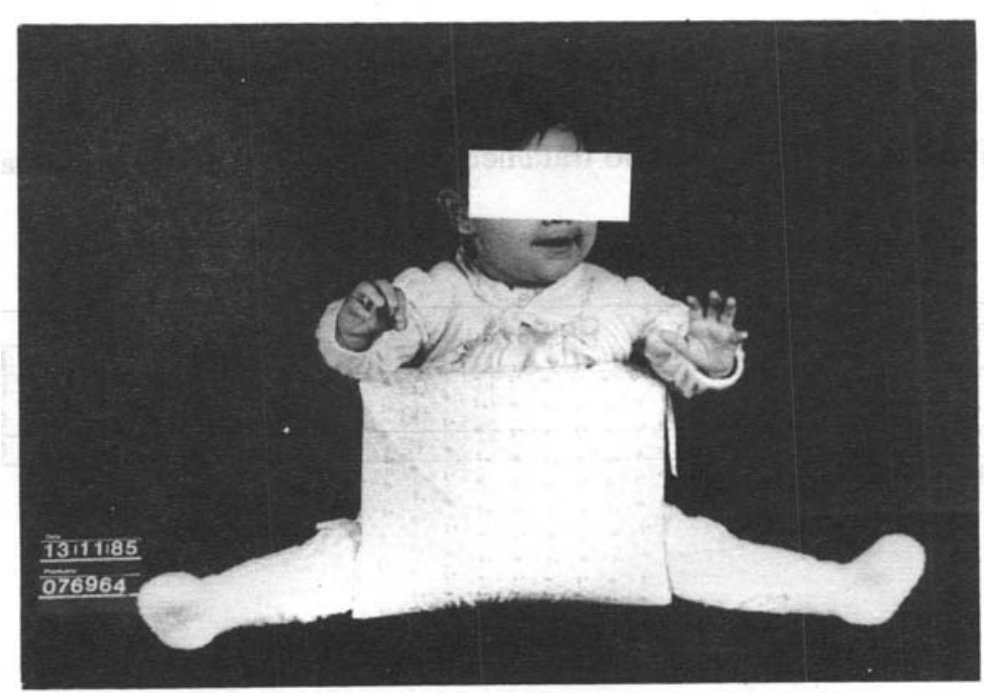

Figura 1 - Fralda de Abdução

FONTE: Arquivo médico do Hospital Sarah

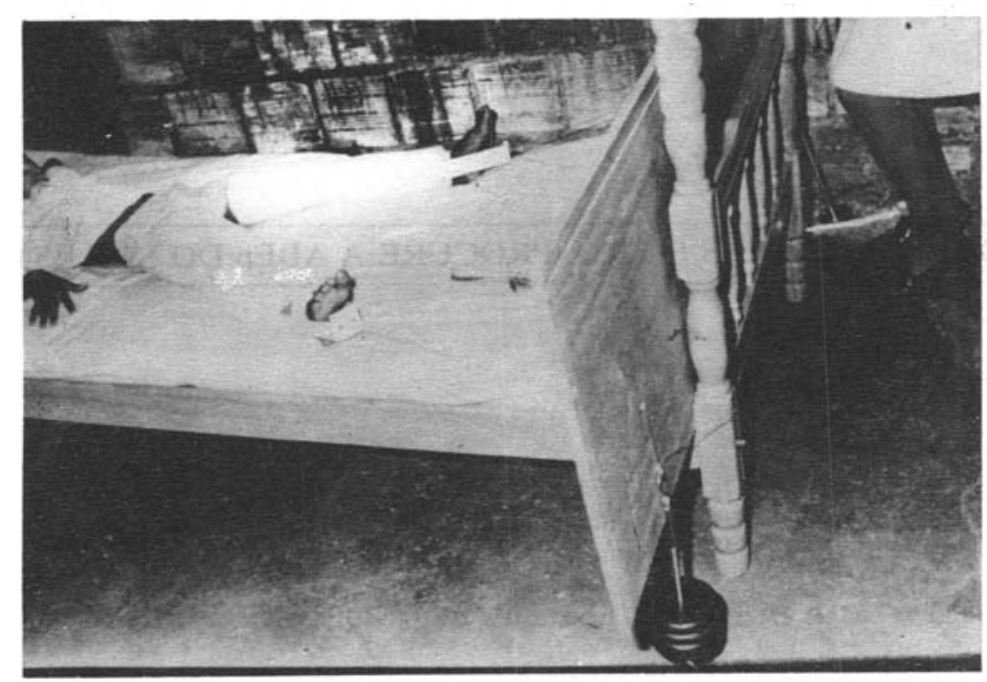

Figura 2 - Tração Cutânea Domiciliar

FONTE: Arquivo médico do Hospital Sarah 


\section{ANEXO 3}

Tabela 1 - Distribuição do sexo segundo a idade dos pacientes analisados:

\begin{tabular}{l|c|c}
\hline \multirow{2}{*}{ Idade } & \multicolumn{2}{|c}{$\mathbf{4}$ a $\mathbf{1 0}$ anos } \\
\cline { 2 - 3 } Sexo & No & $\mathbf{\%}$ \\
\hline Masculino & 16 & 76,2 \\
Feminino & 05 & 238 \\
\hline Total & 21 & 100 \\
\hline FONTE. Questıonárı aplicado e prontuárıos
\end{tabular}

FONTE. Questıonárı aplicado e prontuárıos

Tabela 2 - Distribuição da efetividade do tratamento segundo o tipo de patologia:

\begin{tabular}{|c|c|c|c|c|c|}
\hline \multirow[b]{2}{*}{ Resultado } & \multirow{2}{*}{ Grupo } & \multicolumn{2}{|c|}{1} & \multicolumn{2}{|c|}{ II } \\
\hline & & № & $\%$ & № & $x$ \\
\hline $\begin{array}{l}\text { Efetivo } \\
\text { Náo Efetivo }\end{array}$ & & $\begin{array}{l}09 \\
03\end{array}$ & $\begin{array}{l}75 \\
25 \\
\end{array}$ & $\begin{array}{l}08 \\
01\end{array}$ & $\begin{array}{l}88,9 \\
11,1\end{array}$ \\
\hline Total & & 12 & 100 & 09 & 100 \\
\hline
\end{tabular}

NĀO FIQUE APENAS SÓCIO, PROCURE A ABEn DO SEU ESTADO 